\title{
5 points essentiels pour la prise en charge au cabinet
}

\author{
Marie-Claude Hofnera, Pierre-Yves Rodondi ${ }^{\mathrm{b}}$ \\ a Dr, Unité de Médecine des Violences du CHUV, Centre Universitaire Romand de Médecine Légale, Lausanne;
}

${ }^{b}$ Dr, médecine interne générale FMH, Pully

\section{Résumé}

En Europe, la violence conjugale est la première cause de décès ou d'invalidité des femmes de 16 à 44 ans. Il est pourtant rare que cette «pathologie» figure dans nos dossiers et le médecin est souvent désemparé face à ce problème. Pourtant, son impact sur la santé concerne des domaines qui tous concernent le praticien de médecine interne générale et la plupart des spécialistes: lésions physiques, santé mentale, santé sexuelle et reproductive, augmentation du risque de certaines maladies chroniques. Pour aider le médecin dans ces situations, un protocole a été développé au CHUV: le DOTIP. II décrit cinq étapes pour guider l'intervention: 1. Détecter une éventuelle situation de violence; 2 . Offrir un message clair de soutien; 3 . Traiter et organiser le suivi; 4 . Informer des droits et des ressources du réseau; 5 . Protéger en assurant la sécurité de la victime et des enfants. II existe de nombreux services spécialisés qui peuvent apporter un soutien aux patients concernés. Le médecin doit les connaître, car il ne peut et ne devrait pas prendre en charge à lui seul ces situations qui nécessitent une expertise spécifique, d'autant plus que certaines démarches peuvent prendre beaucoup de temps.

Une patiente de 35 ans se présente à la consultation du Dr V. Elle dit que son mari a tenté de l'étrangler et elle a peur d'avoir une lésion au niveau cervical. L'examen clinique est normal. Lorsque le médecin interroge la patiente sur la raison de cet acte violent, elle lui raconte un contexte de séparation difficile. Elle pense être responsable de cette tentative de strangulation, car c'est elle qui a demandé la séparation et son mari ne supporterait pas cela. Le médecin n'explore pas davantage la situation, d'autant plus qu'il n'a jamais suspecté une attitude violente du mari qui est aussi son patient. Deux mois plus tard, la patiente est amenée aux urgences suite à un coup de poignard de son mari, coup qui n'a heureusement juste pas atteint l'artère carotide.

Chaque mois en Suisse 2 femmes sont tuées par leur partenaire ou ex-partenaire ( 5 tentatives d'homicides/ mois) et 1 femme sur 5 est victime de violence physique et/ou sexuelle dans le cadre de sa relation de couple. En Europe, la violence conjugale est la première cause de décès ou d'invalidité des femmes de 16 à 44 ans. Or, malgré la fréquence de cette problé- matique, il est rare que cette "pathologie» figure dans nos dossiers et le médecin est souvent désemparé face à ce problème, la formation dans ce domaine ne figurant pas dans le cursus des études. Pourtant, en 2002, l'Organisation Mondiale de la Santé a décrété la violence problème de santé publique prioritaire.

\section{Des tableaux cliniques multiples}

Les conséquences de la violence sur la santé touchent quatre domaines qui tous concernent le praticien de médecine interne générale, tout comme la plupart des spécialistes.

Les lésions physiques immédiates sont évidentes, les atteintes à la santé mentale sont polymorphes: dépression, anxiété, troubles de l'alimentation et du sommeil, conduites addictives, tentamen. La violence se révèle souvent par des syndromes de douleurs complexes et non spécifiques de la sphère gynécologique, des complications lors de la grossesse et de l'accouchement ou des MST récidivantes. Plus récemment la violence domestique a été identifiée comme un facteur de risque majeur dans les maladies cardio-vasculaires, l'asthme et le diabète.

\section{Les difficultés de la détection}

Alors que les patientes souhaitent être activement interrogées et qu'elles font confiance au milieu médical, ce problème fait rarement partie de notre anamnèse de routine. Ceci peut s'expliquer par l'ignorance de la fréquence et de la gravité du problème, le manque de formation et de connaissance des lois et des ressources locales, mais c'est surtout l'inconfort que provoque chez nous cette problématique: peur de blesser

Définition de la violence de l'Organisation Mondiale de la Santé 2002 [3]

"L'usage délibéré ou la menace de l'usage délibéré de la force physique ou de la puissance contre soi-même, une autre personne ou contre un groupe ou une communauté, qui entraîne ou risque fort d'entraîner un traumatisme, un décès, un dommage moral, un mauvais développement ou une carence." 

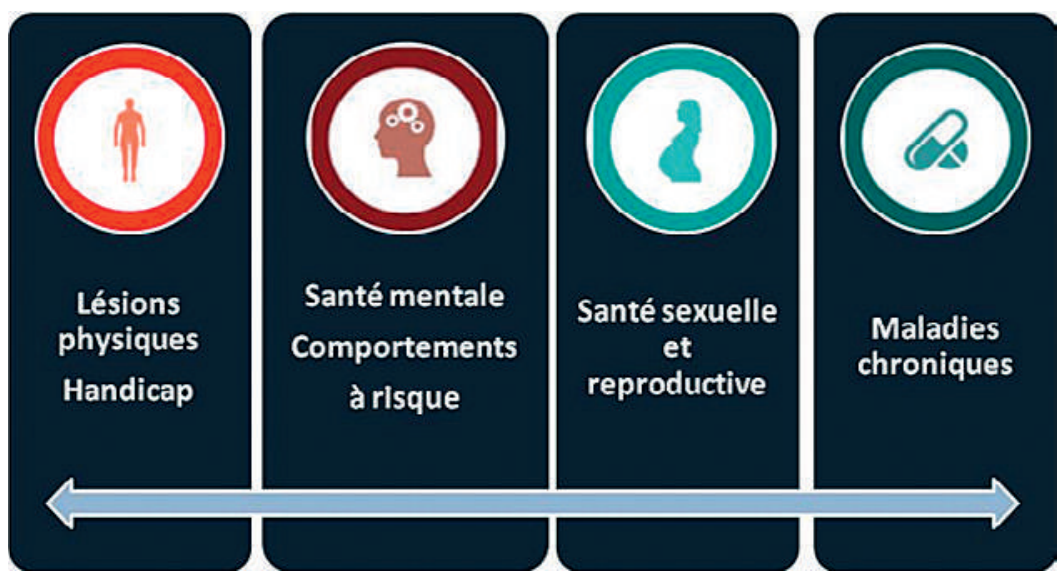

Figure 1: Impact de la violence sur la santé.

par une question que l'on imagine "déplacée», peur de ne pouvoir faire face et répondre de manière adéquate. Disposer d'une marche à suivre et d'informations pratiques nous aide à aller au-devant de nos patients de manière professionnelle.

\section{Comment procéder}

Un protocole a été développé en 2002 au CHUV par une équipe pluridisciplinaire regroupant médecins et personnels infirmiers. Il décrit les cinq étapes qui doivent guider l'intervention:

1. Détecter une éventuelle situation de violence: Penser systématiquement à une violence potentielle et interroger activement ses patients. Chaque patient quel que soit son sexe, son âge, son niveau d'éducation, son origine ou sa religion peut subir de la violence au sein de son couple. Ajouter la violence à son diagnostic différentiel. Poser la question de manière directe, par exemple: «La violence au sein des couples est très fréquente et peut arriver à tout le monde, c'est pourquoi dorénavant je demande à mes patients: comment ça va à la maison?»

2. Offrir un message clair de soutien: La violence est interdite par la loi. La violence est inacceptable quelles que soient les circonstances: «Etre sous l'emprise de l'alcool ne justifie pas que son partenaire agisse de la violence.» Personne ne mérite de subir de la violence, quel que soit son comportement: «Ne pas avoir envie de faire l'amour ne justi-

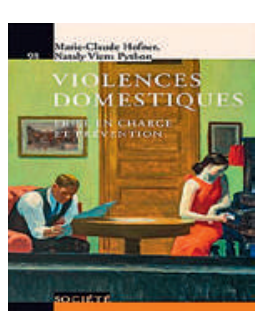

Pour en savoir plus et pour accéder à l'ensemble des données et références citées dans cet article, consulter l'ouvrage: «Violences domestiques. Prise en charge et prévention» de Marie-Claude Hofner et Nataly Viens Python. Coll. Le Savoir Suisse. Presses Polytechniques et Universitaires Romandes; Lausanne, 2014. fie pas une contrainte sexuelle.» La personne n'est pas seule avec son problème, vous êtes capable de l'entendre sans la juger et de respecter ses choix. Vous souhaitez lui offrir de l'aide car vous êtes inquiet pour sa santé.

3. Traiter et organiser le suivi: Apporter les soins somatiques si nécessaires et établir un constat médical selon les règles dans tous les cas, même si un dépôt de plainte n'est pas envisagé (la documentation médico-légale est la seule manière pour une victime de faire valoir le préjudice subi au moment où elle le jugera utile). Tout médecin est habilité à établir un certificat qui doit contenir la description des violences subies (ne pas oublier les menaces et les contraintes) en reprenant les mots du patient (pas d'interprétation), la description des lésions observées (la prise de photo est recommandée), les examens éventuellement entrepris et un état au moment de l'examen y.c. des plaintes du patient (troubles du sommeil, maux de têtes, peur à sortir dans la rue, etc.). Gynécologie Suisse a publié un guide pratique qui présente un modèle pour l'établissement du constat médical [1].

4. Informer de ses droits et des ressources du réseau: La Loi d'Aide aux Victimes d'Infraction (LAVI) garantit les droits des victimes. Des services sont présents dans tous les cantons [2] et offrent des prestations gratuites et en toute confidentialité. Les deux parents impliqués ont le devoir de protéger leurs enfants, qui exposés à la violence de leurs parents sont des victimes de maltraitance. Donner les adresses des ressources locales: police, centre LAVI, services spécialisés, centre d'accueil, etc.

5. Protéger en assurant la sécurité de la victime et des enfants: La personne peut-elle rentrer chez elle sans danger? Si non, orienter vers un centre d'hébergement sécurisé ou demander l'aide de la police. Si la personne souhaite rentrer à la maison, anticiper avec elle la conduite à tenir en cas de nouvelle crise: numéro d'urgence de la police enregistré sur le portable; trousse de secours contenant un peu d'argent pour un taxi, papiers d'identité, carte d'assurance maladie, brochure avec les adresses utiles et un peu de linge; avertir des voisins afin de pouvoir leur confier les enfants et leur demander éventuellement d'appeler la police en cas de crise.

\section{Et les auteurs?}

Si une femme sur cinq subit de la violence la fréquence des personnes ayant recours à la violence dans notre 


\section{A vous d'agir, pensez DOTIP7}

\begin{tabular}{|c|c|c|}
\hline & Je n'y pense pas, parce que... & Sachez que \\
\hline \multirow[t]{2}{*}{$\begin{array}{c}\text { Dépister la violence } \\
\text { conjugale }\end{array}$} & $\begin{array}{l}\text { "Ca n'arrive pas chez les médecins et les avocats" } \\
\text { "ll a l'air si gentil» } \\
\text { "Les hommes violents sont tous des alcooliques" } \\
\text { "Si elle était battue, elle le dirait» }\end{array}$ & $\begin{array}{l}\text { L'OMS recommande un dépistage systématique. } \\
\text { Toute personne peut exercer et subir de la violence, sans distinction de } \\
\text { culture, classe sociale ou éducation. } \\
\text { La honte et la peur font que bien des victimes ne parlent pas } \\
\text { spontanément de la violence subie. }\end{array}$ \\
\hline & Je trouve que ce n'est pas si grave, parce que... & Sachez que \\
\hline \multirow[t]{2}{*}{$\begin{array}{l}\text { Offrir un message } \\
\text { clair de soutien }\end{array}$} & $\begin{array}{l}\text { "Les hommes sont naturellement violents, surtout dans cette culture» } \\
\text { "Ill l'a agressée parce qu'elle l'avait poussé à bout» } \\
\text { "Pour se battre, il faut être deux; la femme est aussi responsable que l'homme» }\end{array}$ & $\begin{array}{l}\text { La violence conjugale est inacceptable. La plupart des actes de violence } \\
\text { sont des délits punissables par la loi. } \\
\text { Toute victime a des droits. La responsabilité des actes de violence } \\
\text { appartient uniquement à leur auteur. }\end{array}$ \\
\hline & Je n'interviens pas, parce que... & Sachez que \\
\hline \multirow[t]{2}{*}{$\begin{array}{l}\text { Traiter } \\
\text { la situation }\end{array}$} & $\begin{array}{l}\text { "Je n'ai pas le temps" } \\
\text { "Je n'ai pas les compétences» } \\
\text { "Je ne sais pas quoi faire» } \\
\text { "J'ai peur d'intervenir, je pourrais être menacé-e en retour» } \\
\text { "C'est un problème privé, cela ne me regarde pas" }\end{array}$ & $\begin{array}{l}\text { Ne pas intervenir, c'est cautionner la violence! } \\
\text { Vous n'êtes pas seul-e, vous pouvez compter sur les autres partenaires du } \\
\text { réseau. } \\
\text { La violence conjugale est un problème de société, de santé et de sécurité } \\
\text { publique. }\end{array}$ \\
\hline & Ca ne sert à rien d'aider les femmes victimes, parce que... & Sachez que \\
\hline \multirow[t]{2}{*}{$\begin{array}{l}\text { Informer la victime } \\
\text { de ses droits et des } \\
\text { ressources du réseau }\end{array}$} & $\begin{array}{l}\text { "Si elles sont battues, c'est qu'elles l'ont cherché ou qu'elles aiment ça» } \\
\text { "Elles retournent toujours avec leur partenaire» } \\
\text { "Elles aussi sont violentes» } \\
\text { "Ce sera encore pire après, surtout pour les enfants» } \\
\text { "De toute façon, il n'y a rien à faire» }\end{array}$ & $\begin{array}{l}\text { Ce n'est pas la violence que les femmes aiment mais leur partenaire, } \\
\text { quand il n'est pas violent. Elles restent ou retournent vers lui pour de } \\
\text { multiples raisons: } \\
\text { espoir, peur, dépendance financière ou au niveau du permis de séjour, } \\
\text { honte, impuissance, isolement, attachement, etc. } \\
\text { La violence conjugale affecte aussi en profondeur la santé et le } \\
\text { développement des enfants qui en sont témoins. }\end{array}$ \\
\hline & Je ne peux rien faire, parce que... & Sachez que \\
\hline $\begin{array}{l}\text { Protéger et prévenir } \\
\text { la récidive }\end{array}$ & $\begin{array}{l}\text { "J'ai déjà tout essayé, elle ne veut pas le quitter" } \\
\text { "Elle a trop peur des représailles, elle m'a fait promettre de ne rien dire» } \\
\text { "Elle refuse d'admettre que ça ne va pas, elle dit qu'elle l'aime encore» }\end{array}$ & $\begin{array}{l}\text { Les victimes ont besoin d'aide pour évaluer le danger et envisager des } \\
\text { scénarios de protection. Subir la violence n'est pas un destin. } \\
\text { Protéger, soutenir et accompagner les victimes est un long processus, } \\
\text { difficile mais possible. }\end{array}$ \\
\hline
\end{tabular}

Dr. Marie-Claude Hofner, Nataly Viens Python, Violence et Maltraitance envers les adultes, Protocole de dépistage et d'intervention, Unité de Prévention, Institut universitaire de médecine sociale et préventive, Lausanne, 2002

Figure 2: Le protocole (DOTIP) est disponible en ligne en français et allemand [4].

patientèle doit elle aussi être élevée. Ces patients ne doivent pas être réduits à la violence qu'ils agissent. Ils sont également en souffrance (40 à $60 \%$ des auteurs ont subi de la violence dans l'enfance) et ont besoin d'écoute et d'orientation. Des services spécialisés existent (www.violencequefaire.ch).

En Europe, la violence conjugale est la première cause de décès ou d'invalidité des femmes de 16 à 44 ans.

\section{En conclusion}

Si l'on reprend la vignette du début, le fait que le médecin connaisse le mari de la patiente a probablement contribué à une certaine gêne à explorer ce comportement violent. Le recours à l'outil «DOTIP» lui aurait notamment permis de dire à la patiente que la violence est interdite par la loi, qu'elle est inacceptable et que seul l'auteur est responsable de son comportement. Le praticien aurait établi un constat, même si sa patiente ne souhaitait pas porter plainte. Il aurait pu l'informer qu'un centre LAVI existait dans son canton, qu'elle pouvait le consulter gratuitement et en toute confidentialité afin d'y trouver un soutien psychologique et juridique. Il aurait pu l'encourager à ne pas hésiter à recourir à la police et l'informer d'autres ressources utiles telles que des centres d'hébergement. La violence domestique est une problématique complexe qui nécessite l'intervention de spécialistes de nombreux domaines vers lesquels il est préférable d'orienter nos patients. Après un bilan initial, le médecin ne peut et ne devrait pas prendre en charge à lui seul ces situations qui nécessitent une formation et une expertise spécifique. De plus, l'information et l'organisation de mesures spécifiques peut prendre beaucoup de temps, dont le médecin traitant ne dispose pas toujours.

\section{Références}

1 Violence domestique guide pratique. Leitfaden häusliche Gewalt Juin 2009. Société suisse de gynécologie et d'obstétrique, Gynécologie Suisse.

2 Liste des centres LAVI suisses. www.sodk.ch/fileadmin/user upload/Fachbereiche/Opferhilfe/Adresslisten/Adressen_der_ OH-Beratungsstellen.pdf

3 Rapport mondial sur la violence et la santé. 2002. Organisation mondiale de la Santé. Genève, Suisse.

4 Hofner MC, Vien Python N. DOTIP Violence conjugale: dépistage, soutien, orientation des personnes victimes. Bureau de l'égalité et de la famille. Fribourg; 2007.

Version française www.fr.ch/bef/fr/pub/violence/informations/ catalogue_de_formation/protocole_dintervention_dotip.htm Version allemande www.fr.ch/bef/de/pub/gewalt/ informationen/weiterbildungskatalog/handbuch_fuer_ fachpersonen_.htm. 